\title{
Comparison of Frequency and Time Domain Schemes for MIMO Broadband Fixed Wireless Access
}

\author{
Pei Xiao ${ }^{1}$, Ioannis Chatzigeorgiou ${ }^{2}$, Rolando Carrasco ${ }^{1}$, Ian Wassell ${ }^{2}$ \\ ${ }^{1}$ School of Electrical, Electronic and Computer Engineering \\ Univ. of Newcastle, NE1 7RU, United Kingdom \\ E-mail: pei.xiao, r.carrasco@ncl.ac.uk \\ ${ }^{2}$ Computer Laboratory, University of Cambridge \\ 15 JJ Thomson Avenue, Cambridge, CB3 OFD, United Kingdom \\ E-mail: ic231,ijw24@cam.ac.uk
}

\begin{abstract}
Broadband fixed wireless access (BFWA) is an ideal solution for providing high data rate communications where traditional landlines are either unavailable or too costly to be installed. In this paper we consider a number of alternative techniques to achieve high data rate and high quality of services requirements in these systems, including orthogonal frequency division multiplexing (OFDM), turbo equalization as well as multiple-input multiple-output (MIMO) techniques. In particular, the frequency domain OFDM scheme and time domain turbo equalization will be studied and compared in a MIMO BFWA context, in an attempt to provide some guidelines on how to design high data rate BFWA applications.
\end{abstract}

Keywords: OFDM, MIMO, equalization, broadband fixed wireless access.

\section{INTRODUCTION}

Broadband fixed wireless access (BFWA) systems enable high data rate communications where traditional landlines are either unavailable or too costly to be installed. These systems also enable operators in a competitive environment to roll-out broadband services in a rapid and cost effective manner of this wireless alternative [1]. In this context, BFWA standardization activities have been performed under the auspices of the IEEE 802.16 working group [2], and the Worldwide Interoperability for Microwave Access (WiMAX) Forum was formed in June 2001 to promote conformance and interoperability of the IEEE 802.16 standard. Both orthogonal frequency division multiplexing (OFDM) and single-carrier solutions have been adopted in IEEE 802.16 standard as two alternatives for BFWA systems operating at 2-11 GHz bands [3].

A BFWA system should be designed to provide high data rate wireless access with wire-line quality. The high requirement for quality arises because it has to compete with cable modems and ADSL approaches which operate over static and non-fading channels and hence are able to provide very good quality. In order to be competitive, the BFWA systems must offer similar data rates to their wire-line counterparts. The problems to be faced in the system design include requirements for low latency and low complexity and support of high transmission bit rates.
One of the limiting factors in outdoor wireless transmission is the multipath channel between the transmitter and the receiver giving rise to intersymbol interference (ISI), which degrades the system performance and limits the maximum achievable data rate. The problem can be tackled by employing OFDM technology [4], which transforms the frequency selective channel into a number of parallel flat fading channels. Another effective remedy to combat the detrimental effects caused by ISI is the use of equalization. Both approaches are studied and compared in this paper. In the latter case, our focus is on the turbo equalization algorithm which combines equalizer and channel decoder in an iterative manner. The existing techniques can be broadly classified into trellis based [5] and filter based [6], [7] approaches. However, they are not suitable for high speed wireless links since their complexity grows drastically with data rate. We propose a space-time turbo equalizer which is especially designed for achieving reliable transmission at high data rate. This paper is organized as follows: Section II and III introduce the systems with OFDM and time domain turbo equalization schemes, respectively. Two schemes will be compared in Section IV and conclusions will be drawn in Section V based on the comparative results.

\section{OFDM SOLUTION}

Here, we consider space-time coded and OFDM based BFWA systems where redundancy spans space and time domains [8]. Fig. 1 depicts the system block diagram, where $N_{T}$ and $N_{R}$ represent the number of transmit and receive antennas, respectively. At the transmitter, the information bits are encoded and interleaved. The mapper maps groups of two bits into one of four QPSK symbols. The space-time processing block further processes the modulation symbols before passing them to the OFDM block. In particular, the space-time processor generates for each particular OFDM sub-carrier a space-time block code (STBC) according to the transmission scheme specified in [9].

Finally, at each transmit antenna chain, complex symbols corresponding to the elements for a particular time slot for the different STBC are imposed onto orthogonal sub-carriers 

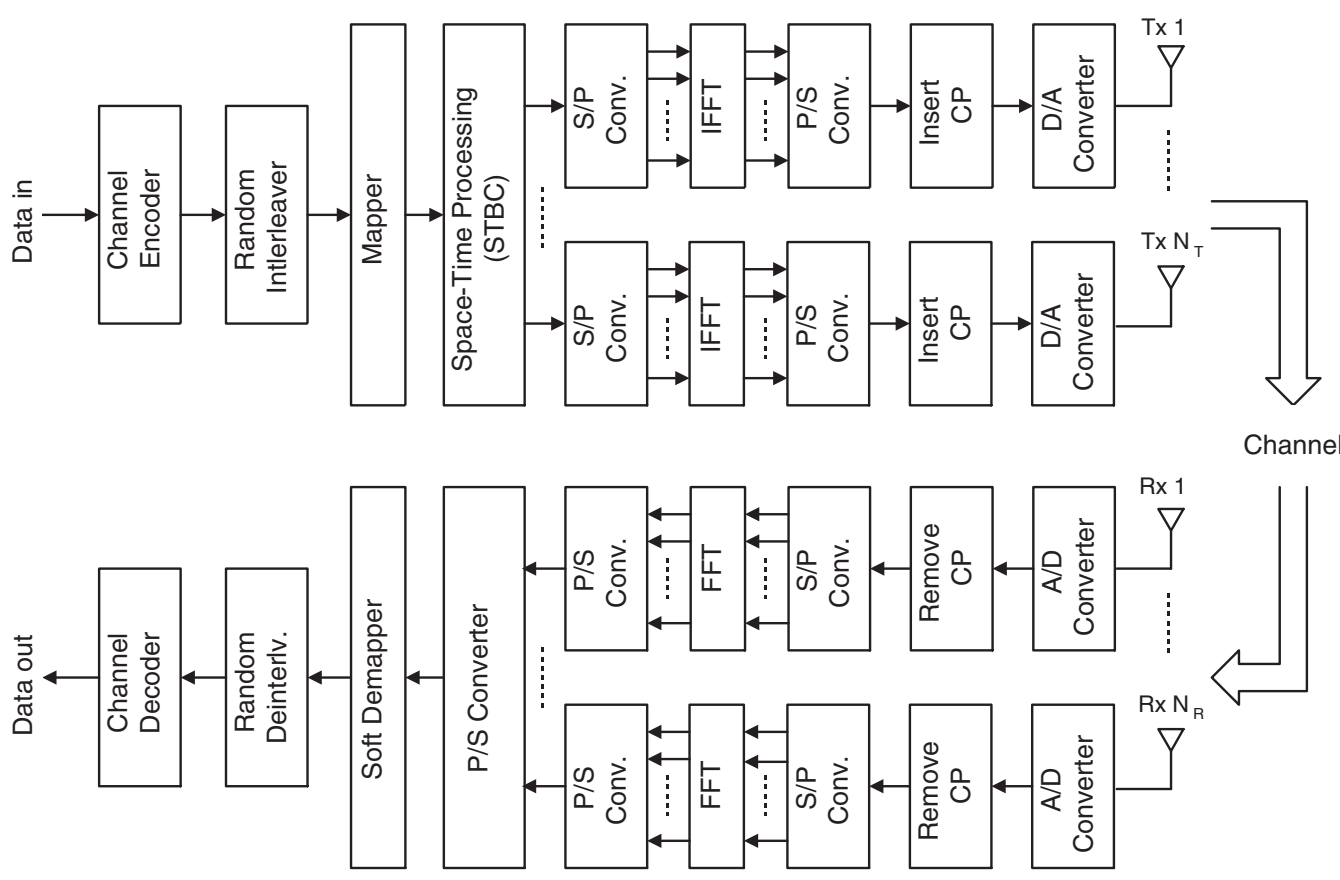

Fig. 1. Block diagram of OFDM based BFWA MIMO system.

by means of an IFFT, a cyclic prefix is inserted with duration longer than the impulse response of the channel to combat intersymbol interference (ISI) and intercarrier interference (ICI), and finally the signal is digital-to-analogue converted.

The OFDM signal is distorted by a BFWA channel as well as AWGN. The BFWA channel has been measured and six Stanford University Interim (SUI) models have been specified for particular scenarios [10], [11]. In this paper we have adopted the SUI-3 model, which corresponds to average British suburban conditions. The line-of-sight (LOS) component is relatively small and the channel is slowly fading as well as mildly frequency selective. We assume that the channel is essentially constant during the transmission of a frame of data. At the receiver, at each receive antenna chain the signal is analogue-todigital converted, the cyclic prefix is removed, and the complex symbols corresponding to the elements for a particular time slot for the different STBC are removed from the orthogonal sub-carriers by means of an FFT. Next, the complex symbols are demapped into soft bits in the form of log-likelihood ratios (LLRs), and the soft bits are then de-interleaved and decoded with the Log-MAP algorithm [12].

\section{TIME DOMAIN TURBO EQUALIZATION}

In this section, we focus on the single-carrier BFWA system and present a space-time turbo equalization algorithm which is well-suited to high data rate BFWA applications. The transmission system under study is shown in Fig. 2. The information sequence $\left\{b_{n}\right\}$ is encoded into coded bits $\left\{u_{n}\right\}$, which are subsequently interleaved and each block of two coded and interleaved bits $u_{n}^{\prime}[0], u_{n}^{\prime}[1]$ is mapped into one of the four QPSK symbols. The interleaver and deinterleaver are denoted as $\Pi$ and $\Pi^{-1}$, in Fig. 2 and Fig. 3, respectively. We use the space-time coding scheme proposed in [9]. The transmitted symbols are space-time encoded according to the generator matrix $\mathbf{G}=\left[\begin{array}{cc}s_{n}^{0} & s_{n}^{1} \\ -s_{n}^{1 *} & s_{n}^{0 *}\end{array}\right]$, where $s_{n}^{0}, s_{n}^{1}$ denote modulation symbols. The operator ()$^{*}$ denotes the conjugate transpose operation when applied to matrices and vectors, and simply the conjugate when applied to scalars.

For simplicity, we assume two transmit antennas and one receive antenna in the derivation of the proposed turbo equalization algorithm. However, its extension to systems with multiple receive antennas is straightforward. Each complex channel coefficient is denoted as $h_{i j}^{l}$ where the first (second) subscript $i(j)$ is the index of the transmit (receive) antenna, the superscript $l$ refers to the number of the channel tap.

In case the transmitted data rate is 2 mega symbols per second ( $2 M$ sysmbols/s), which corresponds to a symbol duration of $T_{s}=0.5 \mu \mathrm{s}$, the multipath fading is modeled as a tapped-delay line with adjacent taps spaced equally at the symbol duration. The received signals at antenna Rx0 during the two symbol periods can be formed as $r_{n}^{0}=h_{00}^{2} s_{n-1}^{0}-h_{00}^{1} s_{n-1}^{1 *}+h_{00}^{0} s_{n}^{0}+$ $h_{10}^{2} s_{n-1}^{1}+h_{10}^{1} s_{n-1}^{0 *}+h_{10}^{0} s_{n}^{1}+w_{n}^{0}$; and $r_{n}^{1}=-h_{00}^{2} s_{n-1}^{1 *}+$ $h_{00}^{1} s_{n}^{0}-h_{00}^{0} s_{n}^{1 *}+h_{10}^{2} s_{n-1}^{0 *}+h_{10}^{1} s_{n}^{1}+h_{10}^{0} s_{n}^{0 *}+w_{n}^{1}$, where $w_{n}^{0}, w_{n}^{1}$ are the complex additive white Gaussian noise with zero mean and variance $N_{0}$. When the data rate is increased to $q \times 2 \mathrm{M}$ symbols/s, and assume $q$ is an even integer value ${ }^{1}$, the received signal is then formed as

$$
\begin{gathered}
r_{n}^{0}=h_{00}^{2} s_{n-q}^{0}+h_{00}^{1} s_{n-\frac{q}{2}}^{0}+\underline{h_{00}^{0} s_{n}^{0}}+h_{10}^{2} s_{n-q}^{1} \\
+h_{10}^{1} s_{n-\frac{q}{2}}^{1}+\underline{h_{10}^{0} s_{n}^{1}}+w_{n}^{0},
\end{gathered}
$$

${ }^{1}$ The received signal is expressed differently when $q$ is odd. However, the principle of the algorithm remains the same. 


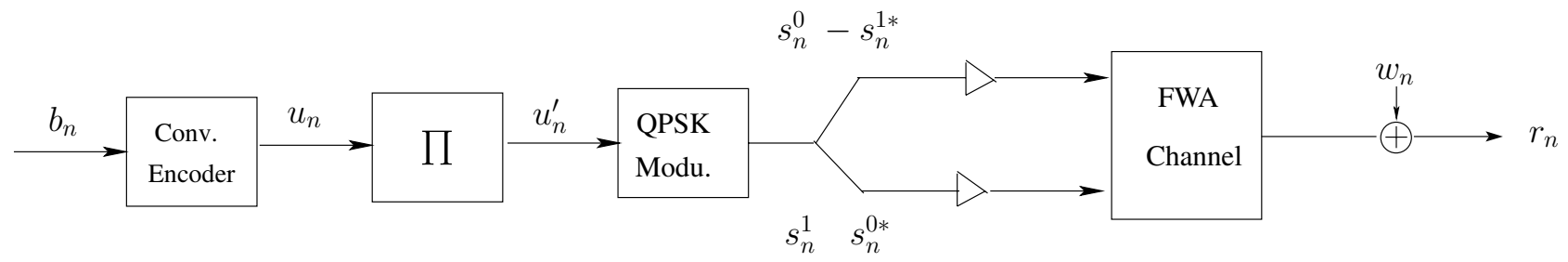

Fig. 2. Block diagram of transmitter for single-carrier STBC coded BFWA system.

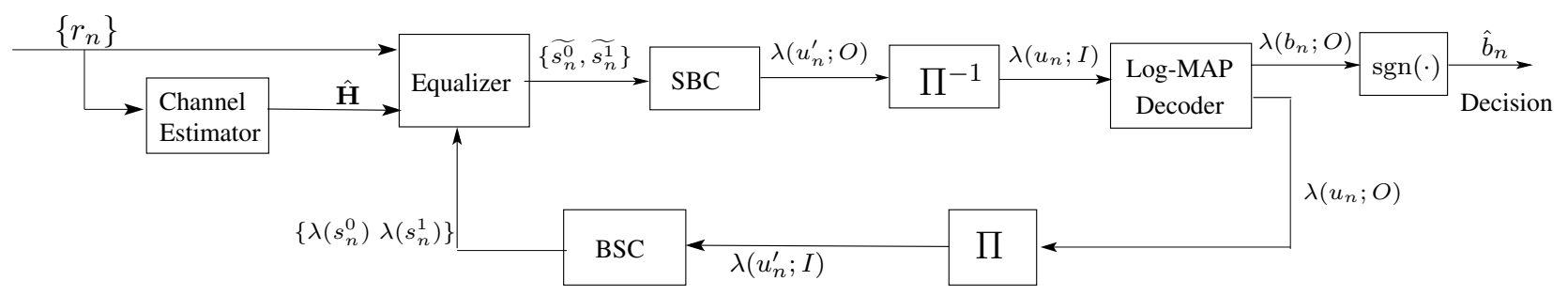

Fig. 3. Block diagram for turbo equalization.

$$
\begin{aligned}
& r_{n}^{1}=- h_{00}^{2} s_{n-q}^{1 *}-h_{00}^{1} s_{n-\frac{q}{2}}^{1 *}-\underline{h_{00}^{0} s_{n}^{1 *}}+h_{10}^{2} s_{n-q}^{0 *} \\
&+h_{10}^{1} s_{n-\frac{q}{2}}^{0 *}+\underline{h_{10}^{0} s_{n}^{0 *}}+w_{n}^{1} .
\end{aligned}
$$

For the symbols of interest $s_{n}^{0}$ and $s_{n}^{1}$ (which are underlined in the above equations so that they can be distinguished from the interference symbols and the noise), the interfering symbols are $s_{n-q}^{0}, s_{n-\frac{q}{2}}^{0}, s_{n-q}^{1}$, etc.. Therefore, the interference does not come from their next neighbouring symbols as in the previous case, and ISI spans for much longer time interval.

The proposed turbo equalization algorithm is illustrated in Fig. 3. First, an estimate of the channel $\hat{h}_{i j}^{l}$ is obtained using a training sequence. In the meantime, a modified Alamouti algorithm is used to obtain the soft values of the transmitted symbols in the form of log-likelihood ratio (LLR) $\left\{\lambda\left(s_{n}\right)=\right.$ $\left.\lambda\left(x_{n}\right)+j \lambda\left(y_{n}\right)\right\}$ where $s_{n}$ denotes either $s_{n}^{0}$ or $s_{n}^{1}$. The channel estimate $\hat{h}_{i j}^{l}$ and symbol estimates $\left\{\lambda\left(s_{n}\right)\right\}$ are passed to the equalizer, which computes $\widetilde{s_{n}}$, the soft decision of $s_{n}$. The soft estimate of the symbol is then mapped to the LLR values of coded bits $\left\{\lambda\left(u_{n}^{\prime} ; O\right)\right\}$ by the symbol-to-bit converter (SBC), which are deinterleaved to yield $\left\{\lambda\left(u_{n} ; I\right)\right\}$. Based on the soft inputs, a Log-MAP decoder computes the LLR for each information bit $\lambda\left(b_{n} ; O\right)$ and each coded bit $\lambda\left(u_{n} ; O\right)$. The former is used to make decisions on the transmitted information bit at the final iteration, and $\lambda\left(u_{n} ; O\right)$ is interleaved and passed through a bit-to-symbol converter (BSC) to derive a soft symbol estimate $\lambda\left(s_{n}\right)$, which is used for equalization at the next iteration. We use the notations $\lambda(\cdot ; I)$ and $\lambda(\cdot ; O)$ to denote the input and output ports of a soft-input and soft-output device. The equalization algorithm will be described in detail next. The focus of this study is the high data rate BFWA applications, for which the received signal is expressed by (1).

\section{A. First coherent combining stage}

We obtain an estimate of the transmitted symbols at the first equalization stage so that interference cancellation can be carried out in the following stages. The Alamouti algorithm was originally developed for flat fading channels and so does not take into consideration the ISI introduced by frequencyselective fading channels. Some modifications have to be made to in order to combat ISI and obtain multipath diversity gain. Let us take pairs of received samples that are $q / 2$-symbol interval apart

$$
\begin{aligned}
& r_{n+q / 2}^{0}=h_{00}^{2} s_{n-q / 2}^{0}+\underline{\underline{h_{00}^{1}} s_{n}^{0}}+h_{00}^{0} s_{n+q / 2}^{0}+h_{10}^{2} s_{n-q / 2}^{1} \\
& +\underline{\underline{h_{10}^{1} s_{n}^{1}}}+\overline{h_{10}^{0} s_{n+q / 2}^{1}}+w_{n+q / 2}^{0}, \\
& r_{n+q / 2}^{1}=-h_{00}^{2} s_{n-q / 2}^{1 *}-\underline{\underline{h_{00}^{1} s_{n}^{1 *}}}-h_{00}^{0} s_{n+q / 2}^{1 *}+h_{10}^{2} s_{n-q / 2}^{0 *} \\
& +h_{10}^{1} s_{n}^{0 *}+\overline{h_{10}^{0} s_{n+q / 2}^{0 *}}+w_{n+q / 2}^{1} ; \\
& r_{n+q}^{0}=\underbrace{h_{00}^{2} s_{n}^{0}+h_{00}^{1}} s_{n+\frac{q}{2}}^{0}+h_{00}^{0} s_{n+q}^{0}+\underbrace{h_{10}^{2} s_{n}^{1}} \\
& +h_{10}^{1} s_{n+\frac{q}{2}}^{1}+h_{10}^{0} s_{n+q}^{1}+w_{n+q}^{0}, \\
& r_{n+q}^{1}=\underbrace{-h_{00}^{2} s_{n}^{1 *}}-h_{00}^{1} s_{n+\frac{q}{2}}^{1 *}-h_{00}^{0} s_{n+q}^{1 *}+\underbrace{h_{10}^{2} s_{n}^{0 *}} \\
& +h_{10}^{1} s_{n+\frac{q}{2}}^{0 *}+h_{10}^{0} s_{n+q}^{0 *}+w_{n+q}^{1} ;
\end{aligned}
$$

From (1) and (2), one can see that the desired symbols $s_{n}^{0}, s_{n}^{1}$ not only appear in the first-tap terms (with one line underneath), but also appear in the second-tap terms (with two lines underneath), as well as in the third-tap terms (with underbrace). In order to take advantage of multipath propagation and obtain diversity gain, we should apply the Alamouti scheme on all the three taps and combine the desired signals from different taps. The three-path combining scheme can be expressed as

$$
\begin{aligned}
\widetilde{s_{n}^{0}}= & \hat{h}_{00}^{0 *} r_{n}^{0}+\hat{h}_{10}^{0} r_{n}^{1 *}+\hat{h}_{00}^{1 *} r_{n+q / 2}^{0}+\hat{h}_{10}^{1} r_{n+q / 2}^{1 *}+\hat{h}_{00}^{2 *} r_{n+q}^{0} \\
& +\hat{h}_{10}^{2} r_{n+q}^{1 *}=\sum_{i, l} \hat{h}_{i 0}^{l *} h_{i 0}^{l} s_{n}^{0}+\epsilon_{n}^{0}=P s_{n}^{0}+\epsilon_{n}^{0} \\
\widetilde{s_{n}^{1}}= & \ldots=\sum_{i, l} \hat{h}_{i 0}^{l *} h_{i 0}^{l} s_{n}^{1}+\epsilon_{n}^{1}
\end{aligned}
$$

where $P=\sum_{i, l} \hat{h}_{i 0}^{l *} h_{i 0}^{l}$ is the total received power from different paths, and $\epsilon_{n}^{0}, \epsilon_{n}^{1}$ can be approximated as complex Gaussian random variables with zero mean and variance $N_{\epsilon}$ which can be computed with noise variance and channel coefficients. The conditional PDFs of $\widetilde{s_{n}^{0}}$ and $\widetilde{s_{n}^{1}}$ are thus derived 
as $f\left(\widetilde{s_{n}^{0}} \mid s_{m}\right)=\frac{1}{\pi N_{\epsilon}} \exp \left(-\frac{\widetilde{\mid s_{n}^{0}}-\left.P s_{m}\right|^{2}}{N_{\epsilon}}\right) ;$ and $f\left(\widetilde{s_{n}^{1}} \mid s_{m}\right)=$ $\frac{1}{\pi N_{\epsilon}} \exp \left(-\frac{\left|\widetilde{s_{n}^{1}}-P s_{m}\right|^{2}}{N_{\epsilon}}\right)$. The LLR values can be derived based on the PDFs which will be explained in the next subsection.

\section{B. The subsequent cancellation stage}

The summation in (3) is carried out over all possible values of $i \in\{0,1\}$, and $l \in\{0,1,2\}$. One can see that this scheme also leads to temporal diversity gain in addition to the spatial diversity gain obtained by the original Alamouti scheme. On the other hand, however, $\epsilon_{n}^{0}, \epsilon_{n}^{1}$ in (3) also contain a lot of ISI terms, which in turn will have a detrimental effect on the overall system performance. To tackle this problem, we can employ the multistage interference cancellation technique to cancel the contribution of the ISI terms. Let us denote $\bar{s}_{n-i}^{0}, \bar{s}_{n-i}^{1}$ as a soft estimate of $s_{n-i}^{0}, s_{n-i}^{1}$ from previous stage $\left(\left\{\bar{s}_{n-i}=\bar{x}_{n-i}+\right.\right.$ $\left.j \bar{y}_{n-i}\right\}$ is computed according to its LLR value as $\bar{s}_{n-i}=$ $\left.\tanh \left[\lambda\left(x_{n-i}\right) / 2\right] / \sqrt{2}+j \tanh \left[\lambda\left(y_{n-i}\right) / 2\right] / \sqrt{2}\right)$. The derivation of the LLRs $\lambda\left(x_{n-i}\right)$ and $\lambda\left(y_{n-i}\right)$ will be explained shortly. To simplify the notation, the iteration (stage) index is omitted whenever no ambiguity arises. Given a channel estimate $\hat{h}_{i j}^{l}$ and symbol estimates $\left\{\bar{s}_{n-i}^{0}, \bar{s}_{n-i}^{1}\right\}$, the ISI canceled version of the received signal $r_{n}^{0}$, denoted as $\bar{r}_{n}^{0}$ can be written according to (1) as

$$
\begin{aligned}
& \bar{r}_{n}^{0}=\left(h_{00}^{2} s_{n-q}^{0}-\hat{h}_{00}^{2} \bar{s}_{n-q}^{0}\right)-\left(h_{00}^{1} s_{n-\frac{q}{2}}^{0}-\hat{h}_{00}^{1} \bar{s}_{n-\frac{q}{2}}^{0}\right)+\underline{h_{00}^{0} s_{n}^{0}} \\
& +\left(h_{10}^{2} s_{n-q}^{1}-\hat{h}_{10}^{2} \bar{s}_{n-q}^{1}\right)+\left(h_{10}^{1} s_{n-\frac{q}{2}}^{1}-\hat{h}_{10}^{1} \bar{s}_{n-\frac{q}{2}}^{1}\right)+\underline{h_{10}^{0} s_{n}^{1}}+w_{n}^{0} .
\end{aligned}
$$

Other ISI canceled versions of the received signals, e.g., $\bar{r}_{n}^{1}, \bar{r}_{n+q / 2}^{0}, \bar{r}_{n+q / 2}^{1}, \bar{r}_{n+q}^{0}, \bar{r}_{n+q}^{1}$ can be formed similarly, i.e., by canceling the contribution from the symbols other than $s_{n}^{0}, s_{n}^{1}$. Using the aforementioned combining technique, the soft decisions of $s_{n}^{0}, s_{n}^{1}$ can now be formed based upon the ISI canceled signals as

$$
\begin{aligned}
\widetilde{s_{n}^{0}}= & \hat{h}_{00}^{0 *} \bar{r}_{n}^{0}+\hat{h}_{10}^{0} \bar{r}_{n}^{1 *}+\hat{h}_{00}^{1 *} \bar{r}_{n+q / 2}^{0}+\hat{h}_{10}^{1} \bar{r}_{n+q / 2}^{1 *}+\hat{h}_{00}^{2 *} \bar{r}_{n+q}^{0} \\
& +\hat{h}_{10}^{2} \bar{r}_{n+q}^{1 *}=\sum_{i, l} \hat{h}_{i 0}^{l *} h_{i 0}^{l} s_{n}^{0}+\varepsilon_{n}^{0}=P s_{n}^{0}+\varepsilon_{n}^{0} \\
\widetilde{s_{n}^{1}}= & \ldots=\sum_{i, l} \hat{h}_{i 0}^{l *} h_{i 0}^{l} s_{n}^{1}+\varepsilon_{n}^{1}=P s_{n}^{1}+\varepsilon_{n}^{1}
\end{aligned}
$$

where $\varepsilon_{n}^{0}, \varepsilon_{n}^{1}$ denote the noise plus cancellation residual. Given correct decision feedback, all the ISI terms will be eliminated. The variance of $\varepsilon_{n}^{0}, \varepsilon_{n}^{1}$ will be much smaller than that of $\epsilon_{n}^{0} \epsilon_{n}^{1}$ in (3), consequently, the BER performance will be greatly improved. In contrast to most linear minimum mean square error (MMSE) based equalizers which involve matrix inversion, the proposed scheme only requires linear processing at the receiver as indicated by (4) and (5). Note that the complexity of the above procedure is not affected by the data rate. When the data rate increases, the interfering symbols become further apart from the desired symbols. However, the number of interfering symbols remain the same. Canceling the symbols that are far away is no more complex than canceling the neighboring symbols. Therefore, the complexity of the proposed scheme does not increase with data rate.

At the beginning of the iterative process, the symbol estimates $\left\{\bar{s}_{n-i}^{0}, \bar{s}_{n-i}^{1}\right\}$ needed for interference cancellation are derived by the three-path combining algorithm expressed by (3). In the following stages, they are obtained from the output of the LogMAP decoder. In what follows, we explain how the LLR values for the coded bits are derived so that the equalization process expressed by (4) and (5) can be carried out.

The combined noise and cancellation residual $\varepsilon_{n}^{0}, \varepsilon_{n}^{1}$ can be approximated as a Gaussian random variable [13] with zero mean and variance $N_{\varepsilon}$, i.e., $\varepsilon_{n} \sim \mathcal{N}\left(0, N_{\varepsilon}\right)$. The conditional PDF is thus derived as

$$
\begin{aligned}
& f\left(\widetilde{s_{n}^{0}} \mid s_{m}\right)=\frac{1}{\pi N_{\varepsilon}} \exp \left(-\frac{\widetilde{\mid s_{n}^{0}}-\left.P s_{m}\right|^{2}}{N_{\varepsilon}}\right) ; \\
& f\left(\widetilde{s_{n}^{1}} \mid s_{m}\right)=\frac{1}{\pi N_{\varepsilon}} \exp \left(-\frac{\widetilde{s_{n}^{1}}-\left.P s_{m}\right|^{2}}{N_{\varepsilon}}\right) .
\end{aligned}
$$

For the QPSK modulated signals, the symbol LLR $\lambda\left(s_{n}\right)=$ $\lambda\left(x_{n}\right)+j \lambda\left(y_{n}\right)$ to bits LLRs $\lambda\left(u_{n}^{\prime}[0]\right), \lambda\left(u_{n}^{\prime}[1]\right)$ mapping rule is simply $\lambda\left(u_{n}^{\prime}[0] ; O\right)=\lambda\left(x_{n}\right) ; \quad \lambda\left(u_{n}^{\prime}[1] ; O\right)=\lambda\left(y_{n}\right)$. Next, we show how the LLR value of $x_{n}$ and $y_{n}$ can be derived from $\widetilde{s_{n}^{0}}$. The LLRs $\lambda\left(u_{n+1}^{\prime}[0]\right), \lambda\left(u_{n+1}^{\prime}[1]\right)$ can be computed similarly based on $\widetilde{s_{n}^{1}}$. Based on (6), the LLR value of $x_{n}$ can be computed as

$$
\lambda\left(x_{n}\right)=\ln \frac{f\left(\widetilde{s_{n}^{0}} \mid x_{n}=+1\right)}{f\left(\widetilde{s_{n}^{0}} \mid x_{n}=-1\right)} \approx \ln \frac{\exp \left(-\widetilde{s_{n}^{0}}-\left.P s_{+}\right|^{2} / N_{\epsilon}\right)}{\exp \left(-\widetilde{s_{n}^{0}}-\left.P s_{-}\right|^{2} / N_{\epsilon}\right)}
$$

$$
\begin{aligned}
& =\frac{P}{N_{\epsilon}} \operatorname{Re}\left\{\left[2 s_{+}^{*} \widetilde{s_{n}^{0}}-P\left|s_{+}\right|^{2}\right]-\left[2 s_{-}^{*} \widetilde{s_{n}^{0}}-P\left|s_{-}\right|^{2}\right]\right\} \\
& \approx \frac{1}{N_{0}} \operatorname{Re}\left\{\left[2 s_{+}^{*} \widetilde{s_{n}^{0}}-P\left|s_{+}\right|^{2}\right]-\left[2 s_{-}^{*} \widetilde{s_{n}^{0}}-P\left|s_{-}\right|^{2}\right]\right\}
\end{aligned}
$$

where $s_{+}$denotes the QPSK symbol corresponding to $\max \left\{f\left(\widetilde{s_{n}^{0}} \mid s_{0}\right), f\left(\widetilde{s_{n}^{0}} \mid s_{3}\right)\right\}$, and $s_{-}$denotes the QPSK symbol corresponding to $\max \left\{f\left(\widetilde{s_{n}^{0}} \mid s_{1}\right), f\left(\widetilde{s_{n}^{0}} \mid s_{2}\right)\right\}$ since the real part of the symbols $s_{0}, s_{3}$ corresponds to 0 , and the real part of the symbols $s_{1}, s_{2}$ corresponds to 1 . The approximation in (8) is due to the assumption that interference is perfectly canceled as iterative process goes on, and under such condition, $N_{\varepsilon} \approx P N_{0}$. Dual maxima rule is used in (7) utilizing the fact that one term usually dominates each sum. The LLR value $\lambda\left(y_{n}\right)$ can be derived similarly.

\section{Simulation Results}

Simulation results are presented in this section to assess and compare the performance of the frequency and time domain schemes presented in the previous sections. We employ a rate $1 / 3$ maximum free distance convolutional code with constraint length 5 and generator polynomials $(25,33,37)_{8}$. During each Monte-Carlo run, the block size is set to 1360 information bits followed by 4 tails bits to terminate the trellis. Four zeros are appended at the end of the bit sequence to make the total number of transmitted bits equal to $2^{12}$. For simplicity, we assume perfect channel estimation. It was shown in [14], [15] that given sufficient pilot symbols, the channel estimation error can be made very small, and compared to the results achieved by assuming perfect channel state information (CSI), the turbo 
equalizer has performance loss within a faction of $1 \mathrm{~dB}$. The coded bits are interleaved by a random interleaver and mapped into QPSK symbols, which are transmitted over the SUI-3 BFWA MIMO channels. This channel model includes three fading taps with delays $0 \mu \mathrm{s}, 0.5 \mu \mathrm{s}$ and $1.0 \mu \mathrm{s}$, with relative powers $0 \mathrm{~dB},-5 \mathrm{~dB}$ and $-10 \mathrm{~dB}$, and with $\mathrm{K}$-factors 1,0 and 0 , respectively. The SUI-3 channel model specifies an antenna correlation coefficient value equal to 0.4 and a Doppler spread per tap equal to $0.4 \mathrm{~Hz}$. The simulation curves are obtained by averaging the simulation results over a minimum of 1000 blocks of transmitted data and after at least 100 errors have occurred. For the OFDM system, we use OFDM/QPSK signals with OFDM symbol duration $T=12.8 \mu s$, cyclic prefix duration $T_{\mathrm{CP}}=3.2 \mu \mathrm{s}$, and $N=1024$ sub-carriers. Therefore, each OFDM sample duration is $T_{s}=\frac{T}{N}=0.0125 \mu \mathrm{s}$. For the studied system with rate $1 / 3$ convolutional code and QPSK modulation, the corresponding data rate is approximately $54 \mathrm{Mbps}$ for the information sequence.

The performance comparison between OFDM and turbo equalization is given in Fig. 4 and Fig. 5 for the $2 \mathrm{Tx}-1 \mathrm{Rx}$ and 2Tx-2Rx BFWA systems, respectively. It is observed that the turbo equalization algorithm converges after 4 stages, beyond which the performance improvement is negligible. This indicates that the latency introduced by the iterative process is moderate. Clearly, it is significant improvement by applying iterative process if we compare the topmost curve representing the performance of one time equalization and decoding with the bottommost curve representing the performance of the proposed turbo equalization upon convergence. We also observe that the gain at each iteration increases with increased SNR. Most significant gains are obtained at first 3 iterations. One can see from both figures that the turbo equalizer outperforms the OFDM scheme after 2 iterations, and the performance gain is more significant in the $2 \mathrm{Tx}-2 \mathrm{Rx}$ system than in the $2 \mathrm{Tx}-1 \mathrm{Rx}$ system. For both OFDM and turbo equalization, the performance gain by adding one receiver antenna is over $5 \mathrm{~dB}$. Here, $E_{b}$ refers to the transmitted bit energy, and is not affected by the number of receive antennas. The gain would be over 2 $\mathrm{dB}$ if we define $E_{b}$ as the received bit energy.

The complexity of the turbo equalization scheme is higher than that of the OFDM scheme due to the iterative process involved. However, for each iteration, it complexity is around $O\left(L^{2}\right)$, where $L$ is the number of channel tap (for 3-tap BFWA channel, $L=3$ ), and is irrelevant to the data rate as analyzed previously. This is in sharp contrast to the existing time domain equalization schemes, the complexity of which becomes unaffordable when the data rate goes too high. With the parameters setting in our simulations, the channel delay spread spans over 100 symbols for a single-carrier system. This would mean a transversal filter with at least 100 taps, and at least several hundreds multiplication operations per data symbol if conventional time domain equalization schemes are to be applied since the number of equalizer taps is on the order of the number of data symbols spanned by the multipath [16]. The proposed turbo scheme is well suited to the BFWA applications since the channel conditions are relatively static in nature and a 3-tap model can adequately describe the

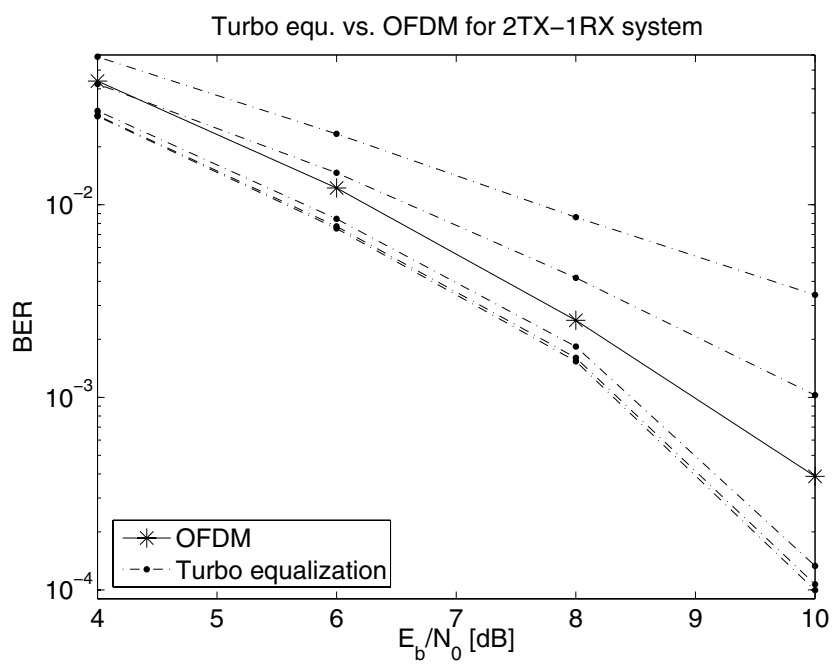

Fig. 4. OFDM vs. Turbo equalization for 2TX-1RX BFWA system. For turbo equalization, the topmost curve represents the initial stage equalization and decoding and bottom curve represents the 5th stage equalization and decoding.

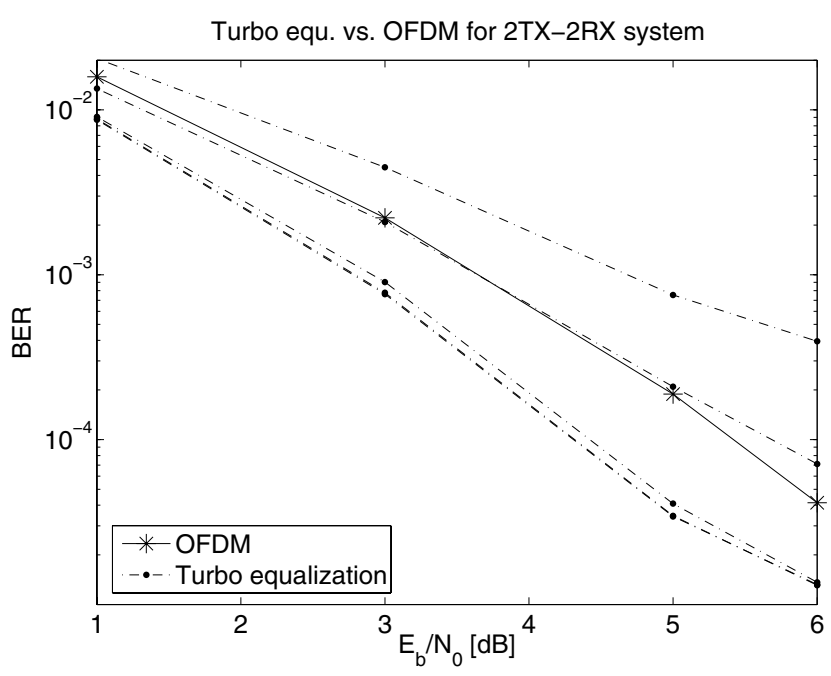

Fig. 5. OFDM vs. Turbo equalization for 2TX-2RX BFWA system.

channel [11], [17]. Complications would arise when extending it to more dynamic systems, such as the mobile WiMAX or a cellular network in which the channels including the number of channel taps are time-varying. The presented turbo scheme would still be applicable if a fixed number of non-zero taps in the equalizer can be allocated adaptively to capture the ISI of the channel, i.e., an equalizer is adaptively assigned with a limited number of taps in order to yield a reduction in complexity in comparison with a conventional sample spaced linear equalizer. However, it might be more advantageous to apply OFDM under such circumstances since the OFDM solution would generally work in spite of the variations in the channel conditions. The complexity of the OFDM scheme is on the order of $\log _{2} N$ multiplications per data symbol [16], and the FFT block length $N$ should be increased as the data rate increases in order to minimize the fraction of overhead due to the insertion of a 
cyclic prefix. Consequently, the complexity gap between the two studied schemes becomes smaller as data rate goes higher.

Another solution to achieve high data rate transmission with low complexity (the same complexity as OFDM scheme) is single carrier frequency domain equalization (SC-FDE) [16], [18]. OFDM was compared with SC-FDE for MIMO BFWA channels in [19]. It was concluded in this paper that OFDM systems perform better than SC-FDE systems using MMSE criterion when the code rate is low $(1 / 2$ and $1 / 3)$. As the code rate increases, e.g., at $2 / 3$, the SC-FDE/MMSE based system begins to outperform the OFDM based system. However, the gap is small in both cases. The curves for SC-FDE are omitted here to conserve space.

\section{Conclusions}

Two feasible solutions for high data rate BFWA applications have been studied and compared in this paper. Numerical results show that both frequency and time domain schemes enable the BFWA system to operate reliably at a high data rate. Comparing to the OFDM scheme, the proposed space-time turbo equalizer achieves better performance at the cost of higher computation complexity incurred by the iterative process. It is especially designed for achieving reliable transmission at high data rate. This is in contrast to the existing time domain equalization algorithms, the complexity of which increases drastically with data rate. OFDM represents a more conservative solution, guaranteeing operation in most environments while not necessarily maximizing performance [20].

\section{REFERENCES}

[1] H. Bolcskei, A. Paulraj, K. Hari, R. Nabar, W. Lu. "Fixed broadband wireless access: state of the art, challenges, and future directions". IEEE Communication Magazine, pp. 100-108, Jan. 2001.

[2] IEEE 802.16 Working Group on Broadband Wireless Access Standards. available at http://grouperieee.org/groups/802/16/. 2002.

[3] C. Eklund. "IEEE Standard 802.16: a technical overview of the wirelessMAN air interface for broadband wireless access". IEEE Communications Magazine, pp. 98-107, June, 2002.

[4] I. Koffman. "Broadband fixed wireless access solutions based on OFDM access in IEEE802.16". IEEE Communication Magazine, vol. 40, pp. 96-103, April, 2002.

[5] C. Douillard, M. Jezequel, C. Berrou. "Iterative correction of intersymbol interference: turbo-equalization". European Transactions on Telecommunications, pp. 507-511, Sept. 1995.

[6] M. Tuchler, R. Koetter, A. Singer. "Turbo equalization: principles and new results", IEEE Transactions on Communications, vol. 50, pp. 754 767, May 2002.

[7] C. Laot, A. Glavieux, Joel Labat. "Turbo equalization: adaptive equalization and channel decoding jointly optimized". IEEE Journal on Selected Areas in Communications, vol. 19, no. 9, Sept. 2001.

[8] D. Agrawal, V. Tarokh, A. Naguib and N. Seshadri. "Space-time coded OFDM for high data-rate wireless communication over wideband channels". Proc. IEEE Vehicular Technology Conference, vol. 3, pp. 2232 2236, May 1998.

[9] A. Alamouti. "A simple transmit diversity technqiue for wireless communications". IEEE Journal on Selected Areas in Communications, vol. 16, no. 8, pp. 1451-1458, Oct. 1998.

[10] Part 16: Air interface for fixed broadband wireless access systems, amendment 2: medium access control modifications and additional physical layer specifications for $211 \mathrm{GHz}, 802.16 \mathrm{a}$ IEEE standard for local and metropolitan area networks, April 2003.

[11] V. Erceg et al. "Channel models for fixed wireless applications". IEEE 802.16a cont. IEEE 802.16.3c-01/29r4, June 2003.

[12] P. Robertson, E. Villebrun, P. Hoeher. "A comparison of optimal and suboptimal MAP decoding algorithms operating in the log domain". Proc. IEEE International Conference on Communications, pp. 1009-1013, 1995.
[13] C. Laot, R. Bidan, D. Leroux. "Low-complexity MMSE turbo equalization: a possible solution to EDGE". IEEE Transactions on Wireless Communications, vol. 4, no. 3. pp. 965-974, May 2005.

[14] P. Xiao, R. Carrasco, I. Wassell. "Estimation of FWA MIMO channels". In proc. IEEE Information Theory Workshop, ITW'2006, pp. 641-645, Oct. 2006.

[15] P. Xiao, R. Carrasco, I. Wassell. "Turbo equalization for space-time block coded FWA systems". In proc. IEEE Information Theory Workshop, ITW'2006, pp. 626-630, Oct. 2006.

[16] D. Falconer, S. Ariavisitakul, A. Benyamin-Seeyar, B. Edison. "Frequency domain equalization for single-carrier broadband wireless systems". IEEE Communications Magazine, pp. 58-66, April 2002.

[17] C. Hong, I. Wassell, G. Athanasiadou, S. Greaves, M. Sellars. "Wideband tapped delay line channel model at $3.5 \mathrm{GHz}$ for broadband fixed wireless access system as a function of subscriber antenna height in suburban environment". The Fourth International Conference on Information, Communications \& Signal Processing (ICICS-PCM 2003), Dec. 2003.

[18] N. AlDhahir. "Single carrier frequency domain equalization for spacetime blockc oded transmissions over frequencyselective fading channels". IEEE Comm. Letters, vol. 5, pp. 304-306, July 2001.

[19] I. Chatzigeorgiou, M. Rodrigues, I. Wassell, R. Carrasco. "Turbo coded OFDM/SC-FDE techniques for MIMO BFWA channels". In Proc. International Symposium on Broadband Communications (ISBC), Dec. 2004.

[20] W. Webb. "Broadband fixed wireless access as a key component of the future integrated communications environment". IEEE Communication Magazine, vol. 39, pp. 115-121, Sept. 2001. 